\title{
Resultados del tratamiento quirúrgico de la patología esofágica benigna con cirugía mínimamente invasiva. Experiencia en 483 pacientes*
}

\author{
Drs. ITALO BRAGHETTO M. ${ }^{1}$, OWEN KORN B. ${ }^{1}$, GONZALO CARDEMIL H. ${ }^{1}$, \\ HÉCTOR VALLADARES H. ${ }^{1}$, GONZALO MASIA L. ${ }^{1}$, CARLOS MANDIOLA B. ${ }^{1}$ \\ Departamento de Cirugía Hospital Clínico de la Universidad de Chile. \\ Santiago, Chile.
}

\begin{abstract}
Minimally invasive surgery for benign esophageal diseases. Experience in 483 patients

Background: Minimally invasive surgical techniques are increasingly used for the treatment of benign esophageal diseases. Aim: To report the results of minimally invasive surgical techniques among patients with benign esophageal diseases. Material and Methods: Four hundred eighty three patients aged 37 to 81 years (184 males), were studied. Of these, 278 had a Barret esophagus, 125 had a hiatal hernia, 75 had achalasia and five had esophageal diverticula. All patients were studied using standard protocols, operated using minimally invasive techniques and followed, registering postoperative complications and recurrence of symptoms. Results: Among patients with esophageal reflux, $85 \%$ had successful results on the long term. Among patients with hiatal hernia and subjected to laparoscopic surgery, $12 \%$ had complications and $12 \%$ had recurrence of symptoms which increased to $17 \%$ if a mesh was not used. The recurrence rate among patients with achalasia is less than 5\%. There was no recurrence among patients with esophageal diverticula. Conclusions: Minimally invasive surgery for esophageal diseases has good success rates, with a lower incidence of complications than open surgery.
\end{abstract}

Key words: Achalasia, hiatal hernia, Barret, surgery.

\section{Resumen}

Introducción: En este artículo se presenta la experiencia de nuestro grupo de trabajo de los resultados obtenidos en el tratamiento quirúrgico con técnicas mínimamente invasivas en patología esofágica benigna. Material y Método: Se analizan los resultados en pacientes sometidos a cirugía antirreflujo por enfermedad por reflujo gastroesofágico, esófago de Barrett, tratamiento de las hernias hiatales, acalasia esofágica y divertículos esofágicos. Resultados: Los resultados obtenidos por nuestro grupo son comparables a los que señala

*Recibido el 11 de junio de 2012 y aceptado para publicación el 22 de octubre de 2012.

Los autores no refieren conflictos de interés.

Correspondencia: Dr. Italo Braghetto M.

Santos Dumont No 999, Santiago, Chile. Fax: 56-2-7370844

ibraghet@redclinicauchile.cl 
la literatura internacional, con mínima morbilidad y sin mortalidad. En pacientes con reflujo gastroesofágico, los resultados a largo plazo presentan una tasa de éxito en el $85 \%$ de los casos. En pacientes con hernia hiatal operados por vía laparoscópica las complicaciones son cercanas al $12 \%$ y la recurrencia es de un $12 \%$ en promedio, pero se eleva al 17\% cuando no se usa malla. En Acalasia por otro lado, la tasa de recurrencia es menor a un 5\%. Los pacientes operados por divertículos esofágicos no presentan recidiva. Conclusiones: Las técnicas de cirugía mínimamente invasiva diseñadas para el abordaje de los diversos tipos de patologías que afectan al esófago, resultan por lo general, altamente factibles de realizar por cuanto reproducen los resultados de la cirugía abierta pero con menor tasa de complicaciones.

Palabras clave: Patología benigna esofágica, laparoscopia, acalasia, reflujo, divertículos.

\section{Introducción}

En los últimos 15 años, la cirugía mínimamente invasiva ha mostrado grandes avances tanto en la técnica quirúrgica y disponibilidad de nuevo instrumental, como en experiencia en procedimientos mínimamente invasivos, lo que ha favorecido la seguridad de los procedimientos y ha disminuido su morbimortalidad. Numerosos son los estudios que se han realizado en el seguimiento de patologías benignas susceptibles de ser abordadas por cirugía mínimamente invasiva, tales como enfermedad por reflujo gastroesofágico (RGE), hernia hiatal, divertículos esofágicos y acalasia, patologías que también en nuestro grupo de trabajo son tratadas quirúrgicamente con procedimientos mínimamente invasivos ${ }^{1-3}$.

En este artículo, se analizan los resultados e indicaciones obtenidos por nuestro grupo en pacientes con patología esofágica benigna sometidos a diferentes técnicas quirúrgicas mínimamente invasivas abordados ya sea por vía laparoscópica o toracoscópica, y se los compara con los resultados publicados en la literatura internacional.

\section{Material y Método}

Se incluyen un total de 483 pacientes (184 hombres, 299 mujeres) con un promedio de edad de 51,2 años (rango entre 37 y 81 años) distribuidos de acuerdo a su patología esofágica y la técnica quirúrgica a la que fueron sometidos (Tablas $1 \mathrm{y}$ 2). Todos estos pacientes se estudiaron de acuerdo a nuestro protocolo de estudio de la patología esofágica, lo que incluye endoscopia, biopsia, manometría, monitoreo de $\mathrm{pH}$ intraesofágico de $24 \mathrm{~h}$ y radiología baritada de acuerdo a protocolos previamente publicados y a las definiciones y parámetros descritos ${ }^{4-6}$. Los procedimientos quirúrgicos empleados por el equipo quirúrgico se muestran en la Tabla 2, de acuerdo a las técnicas descritas ampliamente en publicaciones anteriores para cada una de las patologías incluidas en este estudio ${ }^{7-9}$.

Se analizan los resultados postoperatorios, específicamente complicaciones postoperatorias, mortalidad y resultados alejados evaluados objetivamente tanto del punto de vista clínico (presencia de síntomas), como de controles endoscópicos, manométricos, radiológicos y con monitoreo de $\mathrm{pH}$ intraesofágico de $24 \mathrm{~h}$. Se analiza recurrencia de síntomas (pirosis o regurgitación, disfagia, dolor retro-esternal), hallazgos endoscópicos e histológicos (persistencia o reaparición de esofagitis endoscópica), y hallazgos funcionales esofágicos (manometría o monitoreo de $\mathrm{pH}$ de 24 anormal) y radiológicos, de acuerdo al mismo protocolo preoperatorio.

\section{Resultados}

\section{Enfermedad por Reflujo gastroesofágico (RGE)}

Hemos efectuado fundoplicatura calibrada en 193 pacientes: calibración cardial, cierre de pilares y gastropexia posterior, con éxito sintomático en el $82 \%$ de los pacientes. La cirugía laparoscópica muestra una recurrencia subjetiva de un $18 \%$. La recurrencia objetiva evaluada con manometría esofágica postoperatoria resultó anormal en un 13,4\% de los casos y el monitoreo de $\mathrm{pH}$ intraesofágico de $24 \mathrm{~h}$ mostró persistencia de reflujo ácido en un $18,6 \%$, la endoscopia mostró esofagitis erosiva en un porcentaje similar, siendo estos pacientes en su gran mayoría portadores de esofagitis severa con Barrett (Tabla 3).

\section{Esófago de Barrett (EB)}

Este grupo de pacientes $(\mathrm{n}=85)$ merece un análisis aparte. Hay 3 grupos de pacientes, en 25 de ellos se efectúa calibración cardial y fundoplicatura, y en otros 38 , además de la fundoplicatura, se efectúa un procedimiento de supresión ácida y derivación biliar mediante vagotomía, antrectomía más gastroyeyunostomía en Y de Roux. Los resultados se muestran en la Tabla 4. Se incluye un tercer grupo de pacientes $(\mathrm{n}=22)$ que teniendo esófago de Barrett presentan además obesidad mórbida. En estos pacientes se efectuó una gastrectomía casi 
Tabla 1. Indicaciones de procedimientos a realizar según patología esofágica por vía mini-invasiva tóraco o laparoscópica

\begin{tabular}{|ll|}
\hline Diagnóstico & Téenica quirúrgica \\
\hline Enfermedad por RGE no complicado & Nissen-Rosetti Toupet-calibración cardial \\
\hline Esófago de Barret en pacientes no obesos & Calibración cardial + supresión ácida y derivación biliar \\
\hline Esófago de Barrett en pacientes obesos & Gastrectomía 95\% con gastroyeyunostomía en Y de Roux asa larga \\
Hernia hiatal & Hernioplastía con o sin malla \\
\hline Divertículos mediotorácicos & Toracoscópica \\
Divertículos epifrénicos & Laparoscópica \\
Acalasia & Laparoscópica \\
\hline
\end{tabular}

Tabla 2. Experiencia en patología esofágica no tumoral tratada con cirugía laparoscópica

\begin{tabular}{|lcl|}
\hline Patología & n pacientes & Técnica quirúrgica laparoscópica \\
Esofagitis/Barrett corto & 193 & Calibración cardial + gastropexia posterior + cierre de pilares \\
E. de Barrett largo & 85 & $\begin{array}{l}\text { Calibración cardial sola }(\mathrm{n}=25) \\
\text { Calibración cardial + supresión ácida + derivación biliar }(\mathrm{n}=38) \\
\text { GST 95\% + gastroyeyunostomía Y de Roux asa larga }(\mathrm{n}=22)\end{array}$ \\
$\begin{array}{l}\text { Hernia Hiatal } \\
\text { Acalasia }\end{array}$ & 125 & $\begin{array}{l}\text { Calibración cardial + hiatoplastía } \\
\text { Sin malla }(\mathrm{n}=73) \text { Con malla }(\mathrm{n}=52)\end{array}$ \\
Divertículo mediotorácico & 75 & Cardiomiotomía + fundoplicatura de Dor \\
Divertículo epifrénico & 1 & Diverticulectomía transtorácica \\
Total & 4 & Diverticulectomía + miotomía + fundoplicatura de Dor \\
\hline
\end{tabular}

GST: Gastrectomía subtotal.

Tabla 3. Resultados postoperatorios en pacientes sometidos a cirugía antireflujo $(n=193)$

\begin{tabular}{|lcc|}
\hline Síntomas postoperatorios & Sí & No \\
Manometría & $30 *(17,1 \%)$ & $163(82,9 \%)$ \\
Esfinter gastroesofágico & Incompetente & Normal \\
postoperatorio & $26(13,4 \%)$ & $167(86,5 \%)$ \\
Monitoreo pH de $24 \mathrm{~h}$ & anormal & normal** \\
& $36(18,6 \%)$ & $157(81,3 \%)$ \\
Endoscopia & & \\
Esofagitis & Sí & No \\
& $35(18,1 \%)$ & $158(81,9 \%)$ \\
Radiología (cardias anormal) & 22 & 171 \\
\hline
\end{tabular}

*Recurrencia sintomática. $* *$ Normal $=\%$ time $\mathrm{ph}<4=$ more than $3,4 \%$. Score De Meester $>14,3$. total $(95 \%)$ con gastroyeyunostomía en Y de Roux con la finalidad de tratar ambas patologías dado los buenos resultados reportados recientemente en la literatura ${ }^{10}$. Los operados con fundoplicatura clásica, presenta malos resultados, con alta recurrencia de los síntomas de reflujo, esofagitis endoscópica, incompetencia del esfínter gastroesofágico y reflujo ácido postoperatorio anormal, en comparación a los resultados postoperatorios de los pacientes sometidos a supresión ácida y derivación biliar adicionada a la fundoplicatura.

\section{Hernia Hiatal (HH)}

Hemos operado 125 pacientes, 64 casos de hernia hiatal tipo I, 11 casos de tipo II, 35 casos de tipo III y 15 casos de tipo IV. Complicaciones postoperatorias ocurrieron en 16 pacientes $(12,8 \%)$, siendo las más frecuentes las complicaciones respiratorias, 
Tabla 4. Falla sintomática y objetiva en pacientes con esófago de Barrett sometidos a diferentes procedimientos quirúrgicos por vía laparoscópica

\begin{tabular}{|c|c|c|c|c|c|c|}
\hline & Síntomas & \multicolumn{5}{|c|}{ Recurrencia } \\
\hline & n $\quad(\%)$ & $\begin{array}{c}\text { Radiológica } \\
\text { n }(\%)\end{array}$ & $\begin{array}{c}\text { Endoscópica } \\
\text { n }(\%)\end{array}$ & $\begin{array}{c}\text { Manc } \\
\mathbf{n}\end{array}$ & $\begin{array}{l}\text { métrica } \\
(\%)\end{array}$ & $\begin{array}{l}\text { Test de RGE* } \\
\text { n }(\%)\end{array}$ \\
\hline $\begin{array}{l}\text { Calibraci } \\
(\mathrm{n}=25)\end{array}$ & $10 \quad(40)$ & $(36)$ & $(56)^{* *}$ & 7 & $(28)$ & $(44) * * *$ \\
\hline $\begin{array}{l}\text { Fundopli } \\
(\mathrm{n}=38)\end{array}$ & $\begin{array}{l}\text { n biliar } \\
\qquad 1 \quad(2,7)\end{array}$ & $(2,7)$ & $(2,7)$ & 8 & $(32)$ & $1 \quad(2,7)$ \\
\hline $\begin{array}{l}\text { Gastrecto } \\
(\mathrm{n}=22)\end{array}$ & en $Y$ de Rc & 0 & 0 & 22 & & 2 \\
\hline
\end{tabular}

*Monitoreo de $\mathrm{pH}$ intraesofágico de 24 h. **14 esofagitis erosiva, 4 úlcera esofágica, 1 progresión a displasia. ***p A vs $\mathrm{B}=0,000 ; \mathrm{p}$ B vs $\mathrm{C}=0,049$.

Tabla 5. Experiencia en 125 pacientes con hernia hiatales operados por vía laparocópica. Tipo de hernia y recidiva postoperatoria

\begin{tabular}{|c|c|c|}
\hline Tipo de hernia hiatal & n de pacientes & n de recidivas \\
\hline \multirow[t]{3}{*}{ Tipo I } & 64 & $\rightarrow 4$ \\
\hline & & 1 \\
\hline & & 3 \\
\hline \multicolumn{3}{|l|}{ Tipo II } \\
\hline Tipo III & 35 & 1 \\
\hline Tipo IV & $15-$ & 0 \\
\hline Total & 125 & $14 \quad(11,2 \%)$ \\
\hline
\end{tabular}

Tabla 6. Recurrencia según tipo de hernia con y sin malla de refuerzo

\begin{tabular}{|c|c|c|c|c|c|c|}
\hline \multirow[t]{2}{*}{ Malla } & \multicolumn{4}{|c|}{ Tipo } & \multirow[t]{2}{*}{ Total } & \multirow[t]{2}{*}{ Recurrencia } \\
\hline & I (Rec) & II (Rec) & III (Rec) & IV (Rec) & & \\
\hline Sí & $21--$ & $1 \quad(0)$ & $14 \quad(0)$ & $8 \quad(0)$ & 44 & 0 \\
\hline No & $43 \quad(4)$ & (1) & (4) & (4) & 81 & $14(17,3 \%)^{*}$ \\
\hline Total & $64 \quad(4)$ & (1) & (4) & (5) & 125 & $14(11,2 \%)$ \\
\hline
\end{tabular}

$*(\mathrm{p}=0,03)$.

(atelectasia o derrame pleural) en 8 pacientes, disfagia postoperatoria que necesitó dilatación en 4 pacientes, atonía gástrica transitoria en 2 pacientes, 1 paciente con hemoperitoneo y 1 paciente con hemorragia digestiva alta por úlcera gástrica. No hubo mortalidad postoperatoria en esta serie.

Las recidivas fueron evaluadas en función de un estudio radiológico pre y post operatorio. En total hubo 14 casos de recidiva entre los cuales se encontraban 4 casos de los 64 de hernia hiatal tipo
I, 1 paciente de 11 con hernias tipo II, 4 casos en pacientes con hernia hiatal tipo III y 5 casos de los 15 con hernia hiatal tipo IV. Los pacientes en los cuales se ha usado malla de refuerzo la recurrencia de la hernia es significativamente menor (Tablas 5 y 6$)$.

\section{Acalasia esofágica}

Reportamos una serie de 75 pacientes tratados con técnica de cirugía mínimamente invasiva en 
Tabla 7. Resultados manométricos en el tratamiento quirúrgico de la acalasia esofágica

\begin{tabular}{|lccc|}
\hline Manometría & Antes promedio \pm DS [mmHg] & Después promedio \pm DS [mmHg] & p \\
- Presión EGE & $33,5 \pm 9,09$ & $9,8 \pm 3,5$ & 0,000 \\
- Amplitud ondas & $29,2 \pm 11,3$ & $35,8 \pm 14,2$ & $\mathrm{~ns}$ \\
- Relajación \% EGE & $56,2 \pm 22,3$ & $100 \pm 0,0$ & 0,000 \\
Radiología & Antes promedio \pm DS [mm] & Después promedio \pm DS [mm] & p \\
- EGE & $2,0 \pm 2,5$ & $15,1 \pm 3,9$ & 0,000 \\
- Cuerpo & $51,4 \pm 16,3$ & $30,0 \pm 3,4$ & 0,000 \\
\hline
\end{tabular}

EGE: esfínter gastroesofágico.

quienes se considera una cardiomiotomía anterior laparoscópica asociada a una fundoplicatura de Dor sin disección de estructuras anatómicas periesofágicas laterales y posteriores. En los 75 casos, el diagnóstico fue realizado en base a criterios clínicos, radiológicos, endoscópicos y pruebas funcionales esofágicas. Los resultados clínicos y funcionales se muestran en la Tabla 7, no hemos tenido recurrencias que motivaran reoperaciones y en sólo 3 pacientes hubo necesidad de dilatación hidrostática complementaria. Del total de pacientes, en 4 casos existieron complicaciones intraoperatorias por daño de la mucosa esofágica, siendo suturadas de inmediato en 3 de ellos, con necesidad de conversión sólo en un caso. No se observaron complicaciones postoperatorias y la estadía hospitalaria fue de 3 días. En pacientes con síntomas de RGE postoperatorio, se encontró reflujo ácido positivo, presentando tan sólo uno de ellos signos de esofagitis erosiva al estudio endoscópico. Del total de pacientes operados, sólo en cinco de ellos se ha precisado de un tratamiento de dilatación endoscópica única dada la presencia de disfagia post quirúrgica, con resultados satisfactorios.

\section{Divertículos esofágicos}

Esta patología es bastante rara y hemos tenido la oportunidad de operar algunos casos de divertículos medio torácicos y epifrénicos. En nuestro grupo se trató 1 paciente portador de una fistula esófagobronquial secundaria a un divertículo medio torácico abordado por vía transtorácica, resecando el divertículo y realizando sutura tanto del lado bronquial como del cuello esofágico del divertículo; además 4 pacientes con divertículos epifrénicos, quienes fueron tratados con resección del divertículo y miotomía del esfínter esofágico inferior abordados por vía transhiatal. (Figuras 1 y 2) No hubo complicaciones postoperatorias en estos pacientes.

\section{Discusión}

\section{Enfermedad por Reflujo gastroesofágico}

Los procedimientos mínimamente invasivos en la cirugía antirreflujo son actualmente aceptados como gold standard para el tratamiento de pacientes con esofagitis por RGE. Los resultados de los procedimientos laparoscópicos son absolutamente

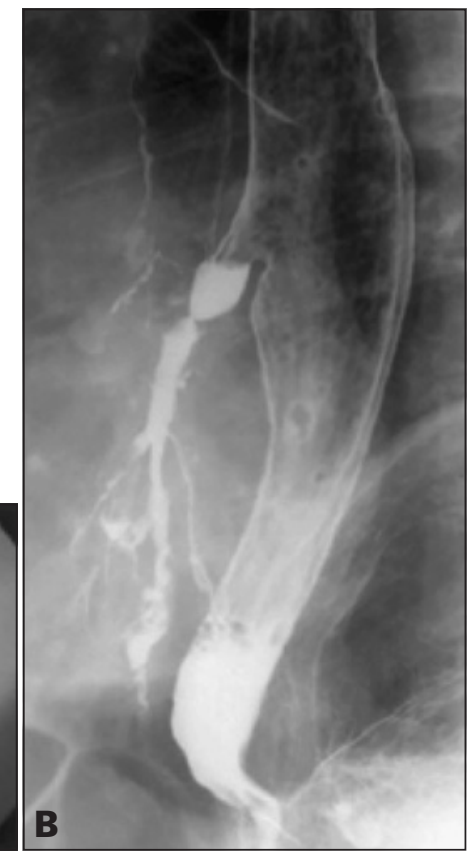

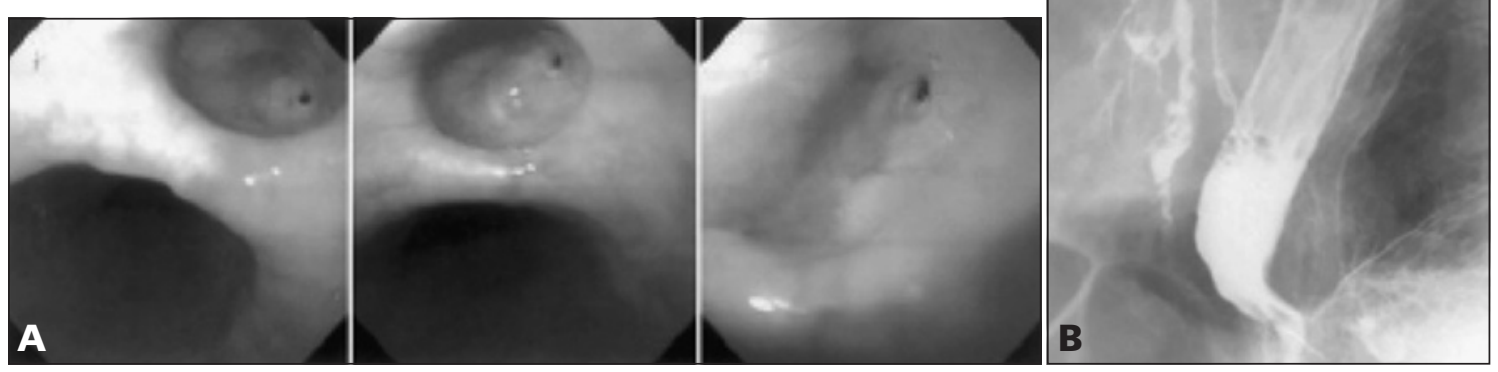

Figura 1. Visión endoscópica (A) y radiológica (B) del divertículo mediotorácico y fístula broncoesofágica. 

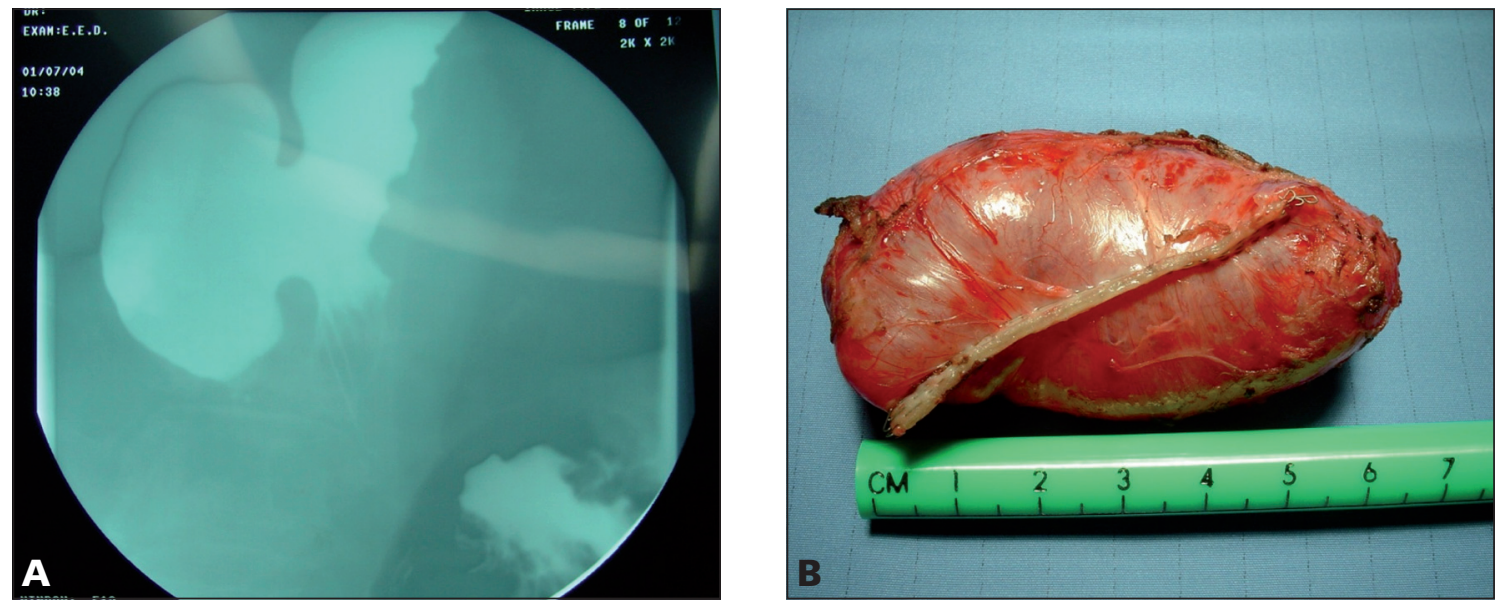

Figura 2. Divertículo epifrénico y pieza resecada por vía laparoscópica transhiatal.

comparables a los obtenidos en la era de la cirugía abierta $^{1-5}$. La fundoplicatura de Nissen, la fundoplicatura modificada de Nissen-Rosetti o la fundoplicatura parcial con las técnicas de Toupet o de Lind, han sido los procedimientos quirúrgicos más comúnmente utilizados $^{6-9}$. En nuestra experiencia la calibración cardial más gastropexia posterior ha sido evaluada a corto y largo plazo, mostrando resultados equivalentes a los otros procedimientos. Si bien estos procedimientos tienen algunas diferencias desde el punto de vista técnico, ambos restablecen la competencia del esfínter esofágico inferior y crean una barrera antirreflujo ${ }^{6}$. Mientras la fundoplicatura de Nissen crea una envoltura completa de unos 3 a $4 \mathrm{~cm}$ alrededor del segmento abdominal del esófago sobre la unión gastroesofágica (UGE), la calibración cardial efectúa una fundoplicatura calibrada restableciendo el diámetro cardial normal, anormalmente dilatado en pacientes con esofagitis por RGE ${ }^{11-13}$.

\section{Esófago de Barrett}

Se ha demostrado que no existen diferencias en relación a los síntomas, presencia de esofagitis y satisfacción del paciente entre la cirugía abierta y la cirugía laparoscópica. En más del 75\% de los pacientes con EB no complicado, existen excelentes resultados sintomáticos. Múltiples estudios han demostrado que pacientes con EB tratados quirúrgicamente parecen tener menor incidencia de cáncer esofágico que pacientes tratados medicamente, punto que, sin embargo, es aún controversial. Los pacientes con EB corto tienen excelentes resultados, similares a los resultados obtenidos en pacientes con esofagitis sin EB. Sin embrago, en pacientes con EB extenso o complicado, con úlcera o estenosis sometidos a fundoplicatura de Nissen, la recurrencia es muy frecuente, siendo necesario medicación antirreflujo en un alto porcentaje de ellos, no existiendo diferencias entre los pacientes tratados en forma médica o quirúrgica en relación al desarrollo de complicaciones ácido-pépticas o neoplásicas. Sin embargo, la recurrencia de reflujo ácido persistente en EB complicado es de un $25 \%$ de los pacientes determinado por monitoreo de $\mathrm{pH}$ intraesofágico de 24 h. La incidencia anual de adenocarcinoma esofágico en pacientes con y sin esófago de Barrett asociado fue de un 0,4 y 0,07\% respectivamente ${ }^{14-19}$.

En una revisión de la literatura, se ha reportado que un 2,5\% de pacientes operados por RGE sin esofagitis (grupo control) deben ser sometidos a cirugía de revisión, en cambio un $6,5 \%$ de los pacientes con esofagitis y un $12 \%$ de los pacientes con EB debieron ser reoperados ${ }^{17}$.

De acuerdo a nuestra conducta ${ }^{15}$, de controlar tanto el reflujo ácido como biliar, factores importantes en la patogenia de la enfermedad hemos operado 29 pacientes con EB extenso complicado, realizando una técnica antirreflujo gastroesofágico asociado con un procedimiento de supresión ácida y derivación duodenal (vagotomía + antrectomía + gastroyeyunostomía en Y de Roux) por vía laparoscópica, demostrando ser una técnica factible, sin mayores complicaciones, lo que actualmente está siendo aceptado en la literatura quirúrgica internacional ${ }^{20}$.

En 21 pacientes con EB y obesidad asociada hemos efectuado una resección gástrica $95 \%$, con anastomosis gastroyeyunal en $\mathrm{Y}$ de Roux y un asa de $150-170 \mathrm{~cm}$ de largo (asa alimentaria), con excelentes resultados sintomáticos y desaparición de la esofagitis. Csendes y otras publicaciones, recomiendan esta técnica para estos pacientes resolviendo el problema de su obesidad y de los síntomas derivados del $\mathrm{RGE}^{10,20-24}$. 


\section{Hernias hiatales}

Las hernias hiatales $(\mathrm{HH})$ se clasifican en hernias hiatales de tipo I, II, III y IV, con frecuencias de $90 \%, 4 \%, 5 \%$ y $1 \%$ respectivamente. La hernia hiatal de tipo I o por deslizamiento axial, es aquella en la cual existe un desplazamiento de la unión gastroesofágica (UGE) hacia el interior del mediastino a través del hiato esofágico, con saco herniario. La hernia hiatal de tipo II o paraesofágica, es aquella en la cual existe una herniación del fondo gástrico hacia el interior del mediastino dentro de un saco herniario, manteniendo la posición correcta de la UGE intraabdominal. La hernia hiatal de tipo III, es una hernia mixta en la cual la UGE y una gran porción del estómago ha migrado hacia el interior del mediastino. Por último, la hernia hiatal de tipo IV, es aquella en la cual existe una gran hernia dentro del mediastino que contiene estómago, omento y a veces otras vísceras abdominales.

Existe además otro tipo de hernia que no es propiamente hiatal (hernia diafragmática parahiatal), en la cual el anillo herniario corresponde a un defecto del músculo diafragmático y no a la protrusión de contenido a través del hiato esofágico ${ }^{25-35}$.

La gran mayoría de los pacientes (sobre un 70\%) presentan síntomas de enfermedad por RGE, muchas veces de larga evolución ${ }^{35}$. Previo a toda cirugía vía laparoscópica se realiza un estudio preoperatorio con endoscopia digestiva alta, radiografía de EED, manometría esofágica y monitoreo de $\mathrm{pH}$ intraesofágico de 24 $\mathrm{h}$, de acuerdo al protocolo previamente publicado. La indicación quirúrgica de las hernias hiatales está dada por su gran asociación con enfermedad por RGE (cerca del 70\%), y complicaciones, tales como vólvulo o incarceración del contenido con obstrucción, lo que puede traducirse como disfagia, vómitos, dolor retroesternal, disnea o broncoaspiración. Cuando esto se asocia a hemorragia digestiva alta, se debe sospechar en isquemia, estrangulación o perforación del órgano, que son complicaciones asociadas a una alta morbilidad y mortalidad ${ }^{35}$.

Hashemi y $\operatorname{cols}^{31}$, evaluaron 54 pacientes sometidos a tratamiento quirúrgico de hernia hiatal de tipo III entre los años 1985 y 1998 comparando cirugía abierta vs laparoscópica. Los resultados del se- guimiento sintomático a 24 meses en pacientes operados por cirugía abierta y por vía laparoscópica mostraron ser semejantes para ambas técnicas. El 88\% de los pacientes sometidos a cirugía abierta y el $77 \%$ de los sometidos a cirugía por vía laparoscópica, mostraron estar satisfechos con los resultados del tratamiento. Sin embargo, estudios más recientes reportan excelentes resultados con cirugía laparoscópica una vez que se ha completado la curva de aprendizaje, sin que los resultados se vean afectados por este factor como en los primeros trabajos publicados ${ }^{36}$.

De la revisión de la literatura publicada entre los años 1995 y 2003, en un total de 764 pacientes operados, las complicaciones se desarrollaron en un $18,1 \%(12-28 \%)$, síntomas postoperatorios persistieron en un $13,3 \%(8-21 \%)$ y la recurrencia se desarrolló en el 11,9\% (0-42\%) de los casos. De los tres estudios que utilizaron malla (121 pacientes), sólo uno presentó un 5\% de recurrencias (Tabla 8$)^{31-36}$. Con los resultados actuales surge la interrogante de si en pacientes con $\mathrm{HH}$ asintomáticos es realmente necesario realizar el procedimiento antirreflujo. Ante ello persiste la discusión, pero la evidencia muestra una tendencia hacia el sí, debido a que una gran mayoría de pacientes tiene enfermedad por RGE objetivo y más del $50 \%$ de éstos presentan síntomas de la enfermedad por RGE. Dentro de la misma línea de pensamiento, se ha visto que una disección extensa intraoperatoria favorecería la incompetencia cardial y, por lo tanto, la enfermedad por RGE. Dentro de los fundamentos que van en contra de esta tendencia, estaría el hecho de que algunos autores señalan una mayor morbilidad y disfagia en pacientes operados, aún siendo más del 10 a 20\% inherente al procedimiento ${ }^{35}$.

Tabla 8. Evolución postoperatoria del tratamiento quirúrgico vía laparoscópica de hernia hiatal. Revisión de la literatura ${ }^{31-34}$

\begin{tabular}{|c|c|c|c|c|}
\hline Autor & $\begin{array}{c}n \\
\operatorname{casos}\end{array}$ & $\begin{array}{c}\text { Complicación } \\
(\%)\end{array}$ & $\begin{array}{c}\text { Síntomas } \\
\text { postoperatorios } \\
(\%)\end{array}$ & $\begin{array}{c}\text { Recurrencia } \\
(\%)\end{array}$ \\
\hline Oddsotir & 53 & 20 & 8 & 13 \\
\hline Pitcher & 12 & 25 & 17 & 0 \\
\hline Luketich & 83 & - & - & 17 \\
\hline Trus & 76 & 28 & - & $5^{*}$ \\
\hline Casabella & 15 & 20 & - & $0 *$ \\
\hline Willekes & 30 & 27 & - & 0 * \\
\hline Perdikis & 65 & 14 & 8 & 8 \\
\hline Mattar & 187 & - & - & 17 \\
\hline Gantert & 35 & 16 & - & 3 \\
\hline $\mathrm{Wu}$ & 38 & 16 & 21 & 23 \\
\hline Hashemi & 54 & 12 & 12 & 15 \\
\hline Díaz & 116 & 13 & 14 & 42 \\
\hline Total & 764 & 18,1 & 13,3 & 11,9 \\
\hline
\end{tabular}

*Con uso de malla. 
El uso de malla como parte de la técnica quirúrgica es controversial. Existe tendencia hacia el uso de ella en base a que existe una mayor recurrencia de $\mathrm{HH}$ en los grupos de pacientes en que no se ha utilizado (19\%) versus los grupos en que sí se ha utilizado (0$5 \%$ ). Por otro lado, existe reticencia de utilizar malla por el hecho de ser un cuerpo extraño no exento de potenciales complicaciones como erosión, fibrosis y disfagia. Probablemente una de sus principales indicaciones es su uso en grandes hernias con cierre a tensión del orificio hiatal. Para evitar dichas complicaciones se aconseja el uso de mallas biológicas o mallas parcialmente reabsorbibles ${ }^{36}$.

La cirugía laparoscópica de $\mathrm{HH}$ ha mostrado ser una técnica factible y segura y con escasas complicaciones. El instrumental utilizado debe ser adecuado para garantizar condiciones óptimas en la obtención de resultados clínicos favorables y funcionales. Como resumen, es mandatario el realizar la disección del saco, la reducción de la hernia, el posicionamiento de una porción de esófago intraabdominal, el desarrollo de una hiatoplastía, la utilización de malla cuando sea necesario, y la realización de gastropexia y de corrección quirúrgica antirreflujo (Tablas 9 y 10).

\section{Acalasia esofágica}

Diversos estudios han comparado la eficacia de las terapias médicas respecto a los resultados ofrecidos por las distintas técnicas quirúrgicas en el enfrentamiento de esta enfermedad, observándose pobres resultados tanto con la inyección de toxina botulínica -Botox ${ }^{\circledR}-$, como con los métodos de dilatación endoscópica, al presentar ambos una mayor tasa de recurrencia de la enfermedad en el seguimiento a largo plazo ${ }^{37-40}$. Es así como la cardiomiotomía anterior se ha convertido en el tratamiento más frecuentemente efectuado en pacientes con acalasia esofágica, existiendo para ello variadas técnicas quirúrgicas que consideran diferencias respecto a vías de abordaje quirúrgico (toracoscópico o laparoscópico), longitud de la miotomía, y asociación o no de técnicas de fundoplicatura.

La cardiomiotomía anterior vía laparoscópica es el tratamiento de elección de la acalasia esofágica, al ser más efectiva en mejorar la disfagia y presentar menores tasas de reflujo gastroesofágico postoperatorio, mínimas tasas de complicaciones intraoperatorias y conversión, y un menor tiempo de estadía intrahospitalaria ${ }^{41-44}$.
Tabla 9. Cirugía laparoscópica: Revisión de 20 trabajos seleccionados

\begin{tabular}{|c|c|c|}
\hline Año & & $1997-2005$ \\
\hline Pacientes & & 1.415 (33 - 203 pacientes incluidos) \\
\hline Conversión & & $3,1 \%(0 *-9,1 \%)$ \\
\hline Edad & & 65,7 años $(18-102)$ \\
\hline Estadía hospitalaria & & 2,7 días $(2-4)$ \\
\hline Cirugía anti RGE & & $94 \%$ \\
\hline Uso de malla & $\begin{array}{l}\text { No } \\
\text { Sí }\end{array}$ & $\begin{array}{r}9 \text { autores } \\
11 \text { autores }\end{array}$ \\
\hline Resección saco & & $90 \%$ \\
\hline
\end{tabular}

*3 Trabajos.

Tabla 10. Complicaciones post operatorias en cirugía laparoscópica de las hernias hiatales: revisión de la literatura

\begin{tabular}{|lc|}
\hline Intraoperatorias & $5,3 \%(0-10 \%)$ \\
\hline Postoperatorias ** & $12,7 \%(0-45 \%)$ \\
Reoperación & \\
- Precoz & $1,2 \%$ \\
- Tardías (>30 días) & $3,0 \%$ \\
Mortalidad & $1,1 \%(15 / 1.366$ pacientes $)$ \\
- Perforación o necrosis & 10 pacientes \\
- Complic. cardiorrespiratorias & 5 pacientes \\
\hline
\end{tabular}

**Gran porcentaje de complicaciones son menores.

La recurrencia de la enfermedad, hernias hiatales paraesofágicas o reflujo gastroesofágico asociado o no a esofagitis, en el postoperatorio alejado varían entre un 10 a un $25 \%$ de frecuencia ${ }^{39-44}$. Se han reportado trabajos que consideran modificaciones a la técnica antes mencionada con el objeto de evitar o reducir las complicaciones ya descritas $^{44}$.

Cabe señalar que las técnicas de cirugía mínimamente invasiva, en este caso la cardiomiotomía anterior asociada a una fundoplicatura de Dor vía laparoscópica sin disección de estructuras periesofágicas laterales y posteriores, en el tratamiento de la acalasia esofágica resultan ampliamente efectivas, reduciendo el riesgo de complicaciones intra y postoperatorias, como así también el riesgo de reflujo gastroesofágico, previniendo posibles factores asociados a tal complicación y disminuyendo la estadía hospitalaria junto a una favorable evolución postquirúrgica.

\section{Patología diverticular del esófago}

La patología diverticular del esófago considera la existencia de divertículos de Zenker; divertículos medio torácicos y divertículos epifrénicos.

En los pacientes con divertículo de Zenker la técnica quirúrgica abierta clásicamente realizada corresponde a 
una diverticulectomía a través de una cervicotomía lateral izquierda con miotomía cricofaríngea cuyos resultados han exhibido tasas de resolución de los síntomas superior a un $90 \%{ }^{45,48-52}$. Las complicaciones varían entre un 10 a un $30 \%$, asociándose a una mortalidad mínima ${ }^{45-53}$.

La cirugía mínimamente invasiva se ha planteado como una alternativa para el enfrentamiento terapéutico de esta patología. Las técnicas de cirugía mínimamente invasiva básicamente consisten en procedimientos endoscópicos, cuyo fin se centra en realizar una división del septum interpuesto entre el divertículo y el esófago cervical, para así establecer una cavidad común entre ambos, además de una miotomía del esfínter esofágico superior ${ }^{44-46}$. Este procedimiento puede ser logrado por medio de técnicas de electrocauterización, uso de láser, o bien, en tercer término, en base a la utilización de sutura mecánica. Chang y $\operatorname{cols}^{46}$, reportó los resultados de la diverticulostomía endoscópica por medio del uso de stapler con una tasa promedio de complicaciones de un $5,5 \%$, y una tasa de mortalidad promedio de un $0,2 \%$. Así también, se observa que el promedio de recurrencia de la sintomatología en los procedimientos abiertos es de $5 \%$, siendo de un 6,6\% para el grupo de técnicas endoscópicas en pacientes seleccionados con indicaciones muy precisas ${ }^{46-53}$.

En cuanto a la enfermedad diverticular que compromete el tercio medio y distal del esófago, en general, los divertículos medio torácicos se acepta que pueden deberse, desde el punto de vista fisiopatológico, a tracción de adherencias antiguas o a propulsión secundaria a alteraciones motoras del órgano, que en el caso de divertículos grandes se asocian a disfagia y regurgitación orofaríngea, con el riesgo de la generación de una eventual fístula hacia la vía aérea (fístula esófago-bronquial) ${ }^{54}$. En tales casos la indicación quirúrgica corresponde a una diverticulectomía realizada por vía transtorácica. En el caso de los divertículos epifrénicos, dada su asociación a acalasia esofágica, los sintomáticos grandes tienen indicación de una diverticulectomía transhiatal junto a una miotomía distal, procedimientos realizados por nosotros en un limitado número de casos por la baja incidencia que presentan ${ }^{55}$.

\section{Conclusión}

En definitiva, las técnicas de cirugía mínimamente invasiva diseñadas para el abordaje de los diversos tipos de patologías que afectan al esófago, resultan por lo general altamente factibles de realizar por cuanto reproducen los resultados de la cirugía abierta. Esto, tomando en consideración el éxito observado en un alto número de pacientes que han sido testigos de sus amplios beneficios sin aumentar sus riesgos, observándose complicaciones significativamente menores respecto a las presentadas por sus contrapartes de cirugía abierta.

Es indudable, que para el adecuado uso de las distintas técnicas de cirugía mínimamente invasiva y la maximización de sus ventajas, es imprescindible un correcto estudio y selección de los pacientes, evaluando continuamente los resultados, siendo para ello mandatorio el mejoramiento continuo del entrenamiento y adquisición de destrezas, junto al desarrollo del mejor criterio clínico.

\section{Referencias}

1. Watson DL, Jamieson GG. Antireflux surgery in the laparoscopic era. Br J Surg. 1998; 85:1173-84.

2. Peters JH, Heimbucker J, Kauer WK, Incarbone R, Bremner C, DeMeester TR. Clinical and physiologic comparison of laparoscopic and open Nissen fundoplication. J Am Coll Surg. 1995;180:385-93.

3. Hinder RA, Filipi CJ, Wetscher G, Neary P, DeMeester TR, Perdikys G. Laparoscopic Nissen fundoplication is an effective treatment for gastroesophageal reflux disease. Ann Surg. 1994;220:472-83.

4. Csendes A, Braghetto I, Korn O, Cortés C. Late subjective and objective evaluation of antireflux surgery in patients with reflux esophagitis: analysis of 215 patients. Surgery 1989;105:374-82.

5. Csendes A, Larraín A. Effect of posterior gastropexy on gastroesophageal sphincter pressure and symptomatic reflux in patients with hiatal hernia. Gastroenterology 1972;63:19-20.

6. Csendes A, Miranda M, Espinoza M. Perimeter and location of the muscular gastroesophageal junction or "cardia" in control subjects and in patients with reflux esophagitis or achalasia. Scand J Gastroenterol. 1981;16:951-6.

7. Braghetto I, Korn O, Debandi A, Burdiles P, Valladares H, Csendes A. Laparoscopic cardial calibration and gastropexy for treatment of patients with reflux esophagitis: pathophysiological basis and result. World J Surg. 2005;29:636-44.

8. Braghetto I, Korn O, Burdiles P, Valladares H, Debandi A. Hernias hiatales verdaderas: tratamiento quirúrgico por vía laparoscópica. Rev Chil Cir. 2002;54:628-38.

9. Braghetto I, Korn O, Valladares H, Rodríguez A, Debandi A, Brunet L. Laparoscopic anterior cardiomyotomy plus Anterior Dor fundoplication without division of lateral and posterior periesophageal anatomic structures for treatment of achalasia of the esophagus. Surg Laparosc Endosc Percutan Tech. 2007; 17:369-74.

10. Csendes A. Effect of Gastric Bypass on Barrett's Esophagus and Intestinal Metaplasia of the Cardia in Patients With Morbid Obesity. J Gastrointest Surg. 
2006;10:259-64.

11. Geagea T. Laparoscopic Nissen-Rossetti fundoplication. Surg Endosc. 1994;8:1080-4.

12. Bell RC, Hanna P, Powers B, Sabel J, Kruza D. Clinical and manometric results of laparoscopic parcial (Toupet) and complete (Nissen-Rossetti) fundoplication. Surg Endosc. 1996;10:724-8.

13. Crookes PF, DeMeester TR. Complete and partial laparoscopic fundoplication for gastroesophageal reflux disease. Surg Endosc. 1997;11:613-4.

14. Csendes A. Surgical treatment of Barrett's esophagus: 1980-2003. World J Surg. 2004; 28:225-31.

15. Braghetto I, Csendes A, Puente J, Burdiles P, Korn O. Esófago de Barret. Rev Chil Cir. 1997,49:119 29.

16. Spechler SJ, Lee E, Ahnen D, Hiranio I, Ramírez F, Raufman JP, et al. Long-term outcome of medical and surgical therapies for gastroesophageal reflux disease: follow-up of a randomized controlled trial. JAMA 2001; 285:2331-8.

17. Peters JH, Wang KK. How should Barrett's ulceration be treated? Surg Endosc. 2004;18:338-44.

18. Jamieson GC, France M, Watson DI. Results of laparoscopic antireflux operations in patients who have Barrett's esophagus. Chest Surg Clin N Am. 2002;12:149-55.

19. Farrell TM, Smith CD, Metreveli RE, Johnson AB, Galloway KD, Hunter JG. Fundoplication provides effective and durable symptom relief in patients with Barrett's esophagus. Am J Surg. 1999;78:18-21.

20. Cobey F. Oeschlager B. Complete regression of Barrett's esophagus after Roux-en-Y gastric bypass. Obesity Surg. 2005:15:710-2.

21. Kamolz T, Granderath F, Pointner R. Laparoscopic antireflux surgery: disease-related quality-of-life assessment before and after surgery in GERD patients with and without Barrett's esophagus. Surg Endosc. 2003;17:880-5.

22. Yau P, Watson DI, Devitt PG, Game PA, Jamieson GG. Laparoscopic antireflux surgery in the treatment of gastroesophageal reflux in patients with Barrett's esophagus. Arch Surg. 2000;135:801-5.

23. Csendes A, Braghetto I, Burdiles P. Antireflux surgery, highly selective vagotomy and duodenal switch procedure: postoperative evaluation in patients with complicated and non complicated Barrett's esophagus. Dis Esoph 2000;13:12-7.

24. Mejía-Rivas MA, Herrera-López A, Hernández-Calleros J, Herrera M, Valdovinos MA. Gastroesophageal Reflux Disease in Morbid Obesity: The Effect of Roux-en-Y Gastric Bypass. Obes Surg. 2008;18:1217-24.

25. Wolf BS. Sliding hiatal hernia: the need for redefinition. Am J Roentgent. 1973;117:231-9.

26. Rodefeld MD, Soper NJ. Parahiatal hernia with volvulus and incarceration: laparoscopic repair of a rare defect. J Gastrointest Surg. 1998;2:193-7.

27. Hiebert CA. Massive hiatal hernia in esophageal surgery. In: Pearson C, et al. 2nd ed. New York: Livingsto-
ne-Churchil 2002;279-84.

28. Landreneau RJ, Johnson JA, Marshall JB, Hazerligg SR, Boley TM, Curtis JJ. Clinical spectrum of paraesophageal herniation. Dig Dis Sci. 1992;37:537-44.

29. Wo JM, Branum GD, Hunter JG, Trus TN, Mauren SJ, Wareing JP. Clinical features of type III (mixed) paraesophageal hernia. Am J Gastroent. 1996;91:914-6.

30. Swanstrom LL, Jobe BA, Kinzie LR. Esophageal motility and outcomes following laparoscopic paraesophageal hernia repair and fundoplication. Am J Surg 1999; 177:359-63.

31. Hashemi M, Peters JH, DeMeester TR, Huprich JE, Queck M, Hagen JA, et al. Laparoscopic repair of large type III hiatal hernia: objective follow up reveals high recurrence rate. J Am Coll Surg. 2000;190:553-61.

32. Díaz S, Brunt LM, Klingensmith ME, Frisella PM, Soper NJ. Laparoscopic paraesophageal hernia repair, a challenging operation: medium-term outcome of 116 patients. J Gastrointest Surg. 2003;7:59-67.

33. Van der Peet DL, Kinkenberg-Knol EC, Poza AA, Sietses C, Eijsbouts QA, Cuesta MA. Laparoscopic treatment of large paraesophageal hernias: both excision of the sac and gastropexy are imperative for adequate surgical treatment. Surg Endosc. 2000;14:1015-8.

34. Oddsdottir M. Paraesophageal hernia. Surg Clin N A. 2000;80:1243-52.

35. Mehta MA, Boddy A, Rhodes M. Review of outcome after laparoscopic paraesophageal hiatal hernia repair. Surg Lap Endosc Perc Tech. 2006:16:301-6.

36. Braghetto I, Korn O, Csendes A,Burdiles P, Valladares H, Brunet L. Postoperative Results After Laparoscopic Approach for Treatment of Large Hiatal Hernias: Is Mesh always needed? Is the Addition of an Antireflux Procedure Necessary? International Surg. 2010:95:807.

37. Csendes A, Braghetto I, Henríquez A. Late results of a prospective randomized study comparing forceful dilatation and esophagomyotomy in patients with achalasia. Gut 1989;30:299-304.

38. Zaninotto G, Annese V, Costantini M, Del Genio A, Epifani M, Gatto G, et al. Randomized controlled trial of botulinum toxin versus laparoscopic Heller myotomy for esophageal achalasia. Ann Surg. 2004;239:364-70.

39. Gockel I, Junginger T, Eckardt VF. Effects of pneumatic dilatation and myotomy on esophageal function and morphology in patients with achalasia. Am Surg. 2005;71:128-31.

40. Braghetto I, Csendes A, Burdiles P, Korn O, Valera JM. Current management of achalasia of the esophagus: critical review and clinical experience. Rev Med Chile 2002;130:1055-66.

41. Pellegrini CA, Leichter R, Patti M. Thorascopic esophageal myotomy in the treatment of achalasia. Ann Thorac Surg. 1993;56:680-2.

42. Robinson TN, Galvani CA, Dutta SK, Gorodner MV, Patti MG. Laparoscopic treatment of recurrent dyspha- 
gia following transthoracic myotomy for achalasia. J Laparoendosc Surg Adv Tech. 2003;13:401-3.

43. Csendes A, Braghetto I, Burdiles P, Korn O, Csendes P, Henríquez A. Very late results of esophagomyotomy for patients with achalasia: clinical, endoscopic, histologic, manometric and acid reflux studies in 67 patients for a mean follow up of 190 months. Ann Surg. 2006;243:196-203.

44. Ackroyd R, Watson DI, Devitt PG, Jamieson GG. Laparoscopic cardiomyotomy and partial fundoplication for achalasia. Surg Endosc. 2001;15:983-6.

45. Peracchia A, Bonavina L, Narne S, Segalin A, Antoniazzi L, Marotta G. Minimally invasive surgery for Zenker's diverticulum: analysis of results in 95 consecutive patients. Arch Surg. 1998;133:695-700.

46. Chang CY, Payapilli RJ, Scher RL. Endoscopic staple diverticulostomy for Zenker's diverticulum: review of literature and experience in 159 consecutive cases. Laringoscope 2003;113:957-65.

47. Bonavina L, Bettineschi F, Fontebasso V, Cechetto A. Cricopharyngeal myotomy and stapling: treatment of choice for Zenker's diverticulum. In: Nabeya K, Hanaoka T, Nogami H, Eds. Recent advances in diseases of the esophagus. Tokyo, Japan: Springer-Verlag. 1993;207-11.

48. Aggerholm K, Illum P. Surgical treatment of Zenker's diverticulum. J Laryngol Otol. 1990;104:312-4.

49. Laing MR, Murthy P, Ah-See KW, Cokburn JS. Surgery for pharyngeal pouch: audit of management with short and long-term follow up. J R Coll Surg Edinb. 1995;40:315-8.

50. Witterick IJ, Gullane PJ, Yeung E. Outcome analysis of Zenker's diverticulectomy and cricopharyngeal myotomy. Head Neck 1995; 17:382-8.

51. Laccourreye O, Menard M, Cauchois R, Huart J, Jouffre V, Bresnau D, et al. Esophageal diverticulum: diverticulopexy versus diverticulectomy. Laryngoscope 1994;104:889-92.

52. Bonafede JP, Lavertu P, Wood BG., Eliachar I. Surgical outcome in 87 patients with Zenker's diverticulum. Laryngoscope 1997;107:720-5.

53. Morton RP, Bartley JR. Inversion of Zenker's diverticulum: the preferred option. Head Neck 993;15:253-6.

54. Braghetto I, Cardemil G, Schwartz E, Valladares H, Rencoret G, Estay R, et al. Video thoracoscopic management of middle esophageal diverticulum with secondary bronchoesophageal fistula: report a case. Surg Today 2008:38:1124-8.

55. Braghetto I, Korn O, Valladares H. Divertículos esofágicos: Tratamiento quirúrgico. Gastroenterol Latinoam. 2005;16:122-6. 